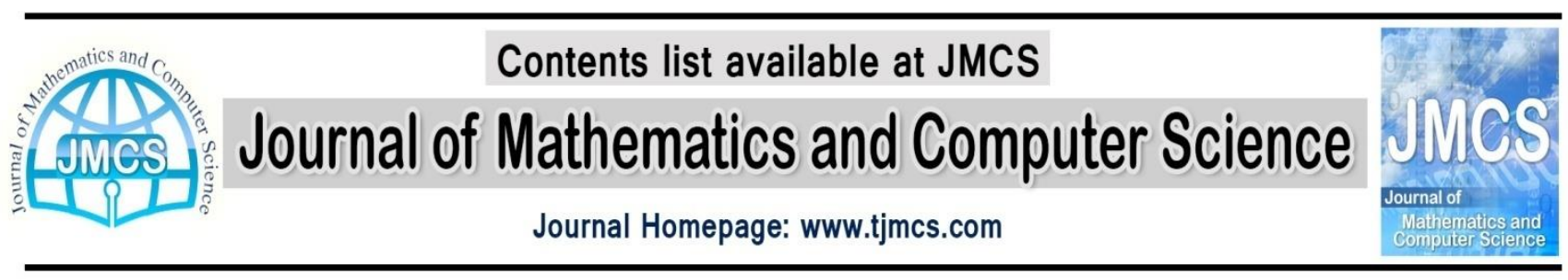

\title{
Dual Smarandache Curves of a Curve lying on Dual Unit Hyperbolic Sphere
}

\author{
Tanju Kahraman ${ }^{1, *}$, Mehmet Önder ${ }^{1,+}$, H. Hüseyin Uğurlu² \\ ${ }^{1}$ Celal Bayar University, Department of Mathematics, Faculty of Arts and Sciences, Manisa, Turkey. \\ *tanju.kahraman@cbu.edu.tr \\ ${ }^{+}$mehmet.onder@cbu.edu.tr \\ ${ }^{2}$ Gazi University, Gazi Faculty of Education, Department of Secondary Education Science and \\ Mathematics Teaching, Mathematics Teaching Program, Ankara, Turkey. \\ hugurlu@gazi.edu.tr
}

Article history:

Received November 2014

Accepted December 2014

Available online January 16, 2015

\section{Abstract}

In this paper, we give Darboux approximation for dual Smarandache curves of spacelike curve on dual unit hyperbolic sphere $\tilde{H}_{0}^{2}$. Firstly, we define the four types of dual Smarandache curves of a dual hyperbolic curve $\tilde{\alpha}(s)$. Then, we obtain the relationships between the dual curvatures of dual hyperbolic curve $\tilde{\alpha}(s)$ and its dual Smarandache curves. Finally, we give an example for Smarandache curves of a spacelike curve on dual unit hyperbolic sphere $\tilde{H}_{0}^{2}$.

MSC 2010: 53A25, 53C40, 53C50.

Key words: E. Study Mapping; Smarandache curves; Darboux approach; Dual unit Hyperbolic sphere.

\section{Introduction}

In the Minkowski spaces, fundamental theory of curves and surfaces is studied and the studies are adapted to other spaces by the many mathematicians $[6,7,8,9,10]$.

In the Minkowski space $E_{1}^{3}$, an oriented timelike line $L$ can be determined by a point $p \in L$ and a normalized direction timelike vector $\vec{a}$ of $L$, i.e. $\|\vec{a}\|=1$. The components of $L$ are obtained by the moment vector $\vec{a}^{*}=\vec{p} \times \vec{a}$ with respect to the origin in $E_{1}^{3}$. The two vectors $\vec{a}$ and $\vec{a}^{*}$ are not 
independent of one another; they satisfy the relationships $\langle\vec{a}, \vec{a}\rangle=-1,\left\langle\vec{a}, \vec{a}^{*}\right\rangle=0$. The pair $\left(\vec{a}, \vec{a}^{*}\right)$ of the vectors $\vec{a}$ and $\vec{a}^{*}$, which satisfies those relationships, is called dual unit timelike vector[2]. Since each dual unit timelike vector corresponds to a timelike line of $E_{1}^{3}$, there is a one-to-one correspondence between the points of a dual unit hyperbolic sphere $\tilde{H}_{0}^{2}$ and the oriented timelike lines of $E_{1}^{3}$. This correspondence is known as E. Study Mapping [2]. As a sequence of that, a differentiable spacelike curve lying fully on dual unit hyperbolic sphere $\tilde{H}_{0}^{2}$ in dual space $D^{3}$ represents a timelike ruled surface which is a surface generated by moving of a timelike line $L$ along a curve $\alpha(s)$ in $E_{1}^{3}$ and has the parametrization $\vec{r}(s, u)=\vec{\alpha}(s)+u \vec{l}(s)$, where $\vec{\alpha}(s)$ is called generating curve and $\vec{l}(s)$, the direction of the timelike line $L$, is called ruling.

In the study of the fundamental theory and the characterizations of space curves, the special curves are very interesting and an important problem. The most mathematicians studied the special curves such as Mannheim curves and Bertrand curves. Recently, a new special curve which is called Smarandache curve is defined by Turgut and Y1lmaz in Minkowski space-time [9]. Then Ali have studied Smarandache curves in the Euclidean 3-space $E^{3}[1]$.

In this paper, we give Darboux approximation for dual Smarandache curves of spacelike curve on dual unit hyperbolic sphere $\tilde{H}_{0}^{2}$. Firstly, we define the four types of dual Smarandache curves of a dual hyperbolic curve. Then, we obtain the relationships between the dual curvatures of dual hyperbolic curve $\tilde{\alpha}(s)$ and its dual Smarandache curves. Finally, we give an example for Smarandache curves of a spacelike curve on dual unit hyperbolic sphere $\tilde{H}_{0}^{2}$.

\section{Preliminaries}

Let $\mathbb{R}_{1}^{3}$ be a 3-dimensional Minkowski space over the field of real numbers $\mathbb{R}$ with the Lorentzian inner product $\langle$,$\rangle given by \langle\vec{a}, \vec{a}\rangle=-a_{1} b_{1}+a_{2} b_{2}+a_{3} b_{3}$, where $\vec{a}=\left(a_{1}, a_{2}, a_{3}\right)$ and $\vec{b}=\left(b_{1}, b_{2}, b_{3}\right) \in \mathbb{R}^{3}$. A vector $\vec{a}=\left(a_{1}, a_{2}, a_{3}\right)$ of $\mathbb{R}_{1}^{3}$ is said to be timelike if $\langle\vec{a}, \vec{a}\rangle<0$, spacelike if $\langle\vec{a}, \vec{a}\rangle>0$ or $\vec{a}=0$, and lightlike (null) if $\langle\vec{a}, \vec{a}\rangle=0$ and $\vec{a} \neq 0$. Similarly, an arbitrary curve $\vec{\alpha}(s)$ in $\mathbb{R}_{1}^{3}$ is spacelike, timelike or lightlike (null), if all of its velocity vectors $\vec{\alpha}^{\prime}(s)$ are spacelike, timelike or lightlike (null), respectively [6]. The norm of a vector $\vec{a}$ is defined by $\|\vec{a}\|=\sqrt{|\langle\vec{a}, \vec{a}\rangle|}$. Now, let $\vec{a}=\left(a_{1}, a_{2}, a_{3}\right)$ and $\vec{b}=\left(b_{1}, b_{2}, b_{3}\right)$ be two vectors in $\mathbb{R}_{1}^{3}$. Then the Lorentzian cross product of $\vec{a}$ and $\vec{b}$ is given by $\vec{a} \times \vec{b}=\left(a_{2} b_{3}-a_{3} b_{2}, a_{1} b_{3}-a_{3} b_{1}, a_{2} b_{1}-a_{1} b_{2}\right)$. By using this definition it can be easily shown that $\langle\vec{a} \times \vec{b}, \vec{c}\rangle=-\operatorname{det}(\vec{a}, \vec{b}, \vec{c})[11]$.

The sets of the unit timelike and unit spacelike vectors are called hyperbolic unit sphere and Lorentzian unit sphere, respectively, and denoted by $H_{0}^{2}=\left\{\vec{a}=\left(a_{1}, a_{2}, a_{3}\right) \in \mathbb{R}_{1}^{3}:\langle\vec{a}, \vec{a}\rangle=-1\right\}$ and $S_{1}^{2}=\left\{\vec{a}=\left(a_{1}, a_{2}, a_{3}\right) \in \mathbb{R}_{1}^{3}:\langle\vec{a}, \vec{a}\rangle=1\right\}$, respectively [11]. 
Let $D=\mathbb{R} \times \mathbb{R}=\left\{\bar{a}=\left(a, a^{*}\right): a, a^{*} \in \mathbb{R}\right\}$ be the set of pairs $\left(a, a^{*}\right)$. For $\bar{a}=\left(a, a^{*}\right)$, $\bar{b}=\left(b, b^{*}\right) \in D$ the following operations are defined on $D$ :

$$
\begin{array}{ll}
\text { Equality } & : \bar{a}=\bar{b} \Leftrightarrow a=b, a^{*}=b^{*} \\
\text { Addition } & : \bar{a}+\bar{b}=\left(a+b, a^{*}+b^{*}\right) \\
\text { Multiplication } & : \bar{a} \bar{b}=\left(a b, a b^{*}+a^{*} b\right)
\end{array}
$$

Then the element $\varepsilon=(0,1) \in D$ satisfies following relationships $\varepsilon \neq 0, \varepsilon^{2}=0, \varepsilon 1=1 \varepsilon=\varepsilon$.

Let consider the element $\bar{a} \in D$ of the form $\bar{a}=(a, 0)$. Then the mapping $f: D \rightarrow \mathbb{R}, f(a, 0)=a$ is an isomorphism. So, we can write $a=(a, 0)$. By the multiplication rule we have that $\bar{a}=a+\varepsilon a^{*}$. Then $\bar{a}=a+\varepsilon a^{*}$ is called dual number and $\varepsilon$ is called dual unit. Thus the set of dual numbers is given by $D=\left\{\bar{a}=a+\varepsilon a^{*}: a, a^{*} \in \mathbb{R}, \varepsilon^{2}=0\right\}$. The set $D$ forms a commutative group under addition. The associative laws hold for multiplication. Dual numbers are distributive and form a ring over the real number field [4].

Dual function of dual number presents a mapping of a dual numbers space on itself. Properties of dual functions were thoroughly investigated by Dimentberg [3]. He derived the general expression for dual analytic (differentiable) function as follows $f(\bar{x})=f\left(x+\varepsilon x^{*}\right)=f(x)+\varepsilon x^{*} f^{\prime}(x)$, where $f^{\prime}(x)$ is derivative of $f(x)$ and $x, x^{*} \in \mathbb{R}$. This definition allows us to write the dual forms of some well-known functions as follows

$$
\left\{\begin{array}{l}
\cosh (\bar{x})=\cosh \left(x+\varepsilon x^{*}\right)=\cosh (x)+\varepsilon x^{*} \sinh (x), \\
\sinh (\bar{x})=\sinh \left(x+\varepsilon x^{*}\right)=\sinh (x)+\varepsilon x^{*} \cosh (x) .
\end{array}\right.
$$

Let $D^{3}=D \times D \times D$ be the set of all triples of dual numbers, i.e., $D^{3}=\left\{\tilde{a}=\left(\bar{a}_{1}, \bar{a}_{2}, \bar{a}_{3}\right): \quad \bar{a}_{i} \in D, i=1,2,3\right\}$. Then the set $D^{3}$ is called dual space. The elements of $D^{3}$ are called dual vectors [2,4]. Similar to the dual numbers, a dual vector $\tilde{a}$ may be expressed in the form $\tilde{a}=\vec{a}+\varepsilon \vec{a}^{*}=\left(\vec{a}, \vec{a}^{*}\right)$, where $\vec{a}$ and $\vec{a}^{*}$ are the vectors of real space $\mathbb{R}^{3}$. Then for any vectors $\tilde{a}=\vec{a}+\varepsilon \vec{a}^{*}$ and $\tilde{b}=\vec{b}+\varepsilon \vec{b}^{*}$ of $D^{3}$, scalar product and cross product are defined by $\langle\tilde{a}, \tilde{b}\rangle=\langle\vec{a}, \vec{b}\rangle+\varepsilon\left(\left\langle\vec{a}, \vec{b}^{*}\right\rangle+\left\langle\vec{a}^{*}, \vec{b}\right\rangle\right)$ and $\tilde{a} \times \tilde{b}=\vec{a} \times \vec{b}+\varepsilon\left(\vec{a} \times \vec{b}^{*}+\vec{a}^{*} \times \vec{b}\right)$, where $\langle\vec{a}, \vec{b}\rangle$ and $\vec{a} \times \vec{b}$ are inner product and cross product of the vectors $\vec{a}$ and $\vec{b}$ in $\mathbb{R}^{3}$, respectively.

The norm of a dual vector $\tilde{a}$ is given by $\|\tilde{a}\|=\|\vec{a}\|+\varepsilon \frac{\left\langle\vec{a}, \vec{a}^{*}\right\rangle}{\|\vec{a}\|}, \quad(\vec{a} \neq 0)$.

A dual vector $\tilde{a}$ with the norm $1+\varepsilon 0$ is called dual unit vector. The set of dual unit vectors is given by $\tilde{S}^{2}=\left\{\tilde{a}=\left(\bar{a}_{1}, \bar{a}_{2}, \bar{a}_{3}\right) \in D^{3}:\langle\tilde{a}, \tilde{a}\rangle=1+\varepsilon 0\right\}$ and called dual unit sphere (For details $[2,4,12]$ ). 
The Lorentzian inner product of two dual vectors $\tilde{a}=\vec{a}+\varepsilon \vec{a}^{*}, \tilde{b}=\vec{b}+\varepsilon \vec{b}^{*} \in D^{3}$ is defined by $\langle\tilde{a}, \tilde{b}\rangle=\langle\vec{a}, \vec{b}\rangle+\varepsilon\left(\left\langle\vec{a}, \vec{b}^{*}\right\rangle+\left\langle\vec{a}^{*}, \vec{b}\right\rangle\right)$, where $\langle\vec{a}, \vec{b}\rangle$ is the Lorentzian inner product of the vectors $\vec{a}$ and $\vec{b}$ in the Minkowski 3-space $\mathbb{R}_{1}^{3}$. Then a dual vector $\tilde{a}=\vec{a}+\varepsilon \vec{a}^{*}$ is said to be timelike if $\vec{a}$ is timelike, spacelike if $\vec{a}$ is spacelike or $\vec{a}=0$ and lightlike (null) if $\vec{a}$ is lightlike (null) and $\vec{a} \neq 0$ [10].

The set of all dual Lorentzian vectors is called dual Lorentzian space and it is defined by $D_{1}^{3}=\left\{\tilde{a}=\vec{a}+\varepsilon \vec{a}^{*}: \quad \vec{a}, \vec{a}^{*} \in \mathbb{R}_{1}^{3}\right\}$.

The Lorentzian cross product of dual vectors $\tilde{a}, \tilde{b} \in D_{1}^{3}$ is defined by $\tilde{a} \times \tilde{b}=\vec{a} \times \vec{b}+\varepsilon\left(\vec{a} \times \vec{b}^{*}+\vec{a}^{*} \times \vec{b}\right)$, where $\vec{a} \times \vec{b}$ is the Lorentzian cross product in $\mathbb{R}_{1}^{3}$.

Let $\tilde{a}=\vec{a}+\varepsilon \vec{a}^{*} \in D_{1}^{3}$. Then $\tilde{a}$ is said to be dual timelike (resp. spacelike) unit vector if the vectors $\vec{a}$ and $\vec{a}^{*}$ satisfy the following equations: $<\vec{a}, \vec{a}>=-1$ (resp. $\left.<\vec{a}, \vec{a}>=1\right), \quad<\vec{a}, \vec{a}^{*}>=0$. The set of all dual timelike unit vectors is called the dual hyperbolic unit sphere, and is denoted by $\tilde{H}_{0}^{2}$,

$$
\tilde{H}_{0}^{2}=\left\{\tilde{a}=\left(\bar{a}_{1}, \bar{a}_{2}, \bar{a}_{3}\right) \in D_{1}^{3}:\langle\tilde{a}, \tilde{a}\rangle=-1+\varepsilon 0\right\}
$$

Similarly, the set of all dual spacelike unit vectors is called the dual Lorentzian unit sphere, and is denoted by $\tilde{S}_{1}^{2}$,

$$
\tilde{S}_{1}^{2}=\left\{\tilde{a}=\left(\bar{a}_{1}, \bar{a}_{2}, \bar{a}_{3}\right) \in D_{1}^{3}:\langle\tilde{a}, \tilde{a}\rangle=1+\varepsilon 0\right\}
$$

(For details see [10]).

\section{Dual Representation and Dual Darboux Frame of a Timelike Ruled Surface with Timelike Ruling}

In the Minkowski 3-space $\mathbb{R}_{1}^{3}$, there exists a one-to-one correspondence between the timelike vectors of dual hyperbolic unit sphere $\tilde{H}_{0}^{2}$ and the directed timelike lines of the Minkowski space $\mathbb{R}_{1}^{3}$ [10]. This correspondence is known as E. Study Mapping. By the aid of this correspondence, the geometry of timelike ruled surfaces is represented by the geometry of dual hyperbolic curves lying on the dual hyperbolic unit sphere $\tilde{H}_{0}^{2}$.

Let now $(\tilde{e})$ be a dual hyperbolic curve represented by the dual timelike unit vector $\tilde{e}(u)=\vec{e}(u)+\varepsilon \vec{e}^{*}(u)$. Then, the given dual curve $(\tilde{e})$ corresponding to the timelike ruled surface $\varphi_{e}=\vec{c}(s)+v \vec{e}(s)$ may be represented by $\tilde{e}(s)=\vec{e}+\varepsilon \vec{c} \times \vec{e}$, where $\langle\vec{e}, \vec{e}\rangle=-1, \quad\left\langle\vec{e}^{\prime}, \vec{e}^{\prime}\right\rangle=1$, $\left\langle\vec{c}^{\prime}, \vec{e}^{\prime}\right\rangle=0$ and $\vec{c}$ is the position vector of the striction curve.

The derivatives of the vectors of dual frame $\{\tilde{e}, \tilde{t}, \tilde{g}\}$ of a timelike ruled surface are given as follows, 


$$
\frac{d \tilde{e}}{d \bar{s}}=\tilde{t}, \frac{d \tilde{t}}{d \bar{s}}=\tilde{e}+\bar{\gamma} \tilde{g}, \frac{d \tilde{g}}{d \bar{s}}=-\bar{\gamma} \tilde{t}
$$

and called dual Darboux formulae of timelike ruled surface $\tilde{e}$ (or $\varphi_{e}$ ). Then the dual Darboux vector of the trihedron is $\tilde{d}=-\bar{\gamma} \tilde{e}-\tilde{g}$.

Dual curvature of the ruled surface is

$$
\bar{\gamma}=\gamma+\varepsilon(\delta+\gamma \Delta)
$$

where $\gamma$ is conical curvature, $\Delta=\operatorname{det}\left(\vec{c}^{\prime}, \vec{e}, \vec{t}\right)$ and $\delta=\left\langle\vec{c}^{\prime}, \vec{e}\right\rangle$.

The functions $\gamma(s), \delta(s)$ and $\Delta(s)$ are the invariants of the timelike ruled surface $\varphi_{e}$. They determine the timelike ruled surface uniquely up to its position in the space. For example, if $\delta=\Delta=0$ we have $\vec{c}$ is constant. It means that the timelike ruled surface $\varphi_{e}$ is a timelike cone.

Dual radius of curvature of dual hyperbolic curve (timelike ruled surface) $\tilde{e}(s)$ is can be calculated analogous to common Lorentzian differential geometry of curves as follows

$$
\bar{R}=\frac{\left\|\frac{d \tilde{e}}{d \bar{s}}\right\|^{3}}{\left\|\frac{d \tilde{e}}{d \bar{s}} \times \frac{d^{2} \tilde{e}}{d \bar{s}^{2}}\right\|}=\frac{1}{\sqrt{1-\bar{\gamma}^{2} \mid}}
$$

The unit vector $\tilde{d}_{o}$ with the same sense as the Darboux vector $\tilde{d}=-\bar{\gamma} \tilde{e}-\tilde{g}$ is given by

$$
\tilde{d}_{o}=-\frac{\bar{\gamma}}{\sqrt{\left|1-\bar{\gamma}^{2}\right|}} \tilde{e}-\frac{1}{\sqrt{\left|1-\bar{\gamma}^{2}\right|}} \tilde{g}
$$

It is clear that $\tilde{d}_{o}$ is timelike (or spacelike) if $|\bar{\gamma}|>1$ (or $|\bar{\gamma}|<1$ ). Then, the dual angle between $\tilde{d}_{o}$ and $\tilde{e}$ satisfies the followings,

$$
\left\{\begin{array}{l}
\cosh \bar{\rho}=-\frac{\bar{\gamma}}{\sqrt{\left|1-\bar{\gamma}^{2}\right|}}, \quad \sinh \bar{\rho}=-\frac{1}{\sqrt{\left|1-\bar{\gamma}^{2}\right|}}, \text { if }|\bar{\gamma}|>1, \\
\sinh \bar{\rho}=-\frac{\bar{\gamma}}{\sqrt{\left|1-\bar{\gamma}^{2}\right|}}, \quad \cosh \bar{\rho}=-\frac{1}{\sqrt{\left|1-\bar{\gamma}^{2}\right|}}, \text { if }|\bar{\gamma}|<1 .
\end{array}\right.
$$

where $\bar{\rho}$ is the dual spherical radius of curvature. Hence,

$$
\bar{R}=\left\{\begin{array}{l}
-\sinh \bar{\rho}, \text { if }|\bar{\gamma}|>1, \\
-\cosh \bar{\rho}, \text { if }|\bar{\gamma}|<1,
\end{array} \text { and } \bar{\gamma}=\left\{\begin{array}{l}
\operatorname{coth} \bar{\rho}, \text { if }|\bar{\gamma}|>1 \\
\tanh \bar{\rho}, \text { if }|\bar{\gamma}|<1
\end{array}\right. \text {, }\right.
$$


[7].

\section{Dual Smarandache Curves of a Curve lying on $\tilde{H}_{0}^{2}$}

In this section, we first define the four different types of the Smarandache curves on dual unit hyperbolic sphere in $D_{1}^{3}$. Then by the aid of dual geodesic trihedron (Dual Darboux frame), we give the characterizations of these dual hyperbolic curves (or timelike ruled surfaces). Since dual spherical curves correspond to timelike or spacelike ruled surfaces, the dual Smarandache curves can be also called Smarandache ruled surfaces. Then, using the found results and relationships we study the developable of the corresponding ruled surface and its Smarandache ruled surface.

\subsection{Dual Smarandache $\tilde{e} \tilde{t}$-curves of a curve lying on $\tilde{H}_{0}^{2}$}

Definition 4.1. Let $\tilde{\alpha}=\tilde{\alpha}(\bar{s})$ be a unit speed regular dual curve lying fully on dual unit hyperbolic sphere $\tilde{H}_{0}^{2}$ and $\{\tilde{e}, \tilde{t}, \tilde{g}\}$ be its moving dual Darboux frame. The dual Smarandache curves defined by
i) $\tilde{\alpha}_{1}=\sqrt{2} \tilde{e}+\tilde{t}$
ii) $\tilde{\alpha}_{2}=\tilde{e}+\sqrt{2} \tilde{t}$

are called the dual Smarandache $\tilde{e} \tilde{t}$-curves of hyperbolic curve $\tilde{\alpha}$. Dual curves $\tilde{\alpha}_{1}$ and $\tilde{\alpha}_{2}$ fully lies on $\tilde{H}_{0}^{2}$ and $\tilde{S}_{1}^{2}$, respectively.

Now we can give the relationships between $\tilde{\alpha}$ and its dual Smarandache $\tilde{e} \tilde{t}$-curves as follows.

Theorem 4.1. Let $\tilde{\alpha}=\tilde{\alpha}(\bar{s})$ be a unit speed regular dual hyperbolic curve lying on dual unit hyperbolic sphere $\tilde{H}_{0}^{2}$. Then the relationships between the dual Darboux frames of $\tilde{\alpha}$ and its dual Smarandache $\tilde{e} \tilde{t}$-curves are given by

$$
\text { i) }\left(\begin{array}{l}
\tilde{e}_{1} \\
\tilde{t}_{1} \\
\tilde{g}_{1}
\end{array}\right)=\left(\begin{array}{ccc}
\sqrt{2} & 1 & 0 \\
\frac{1}{\sqrt{\bar{\gamma}^{2}+1}} & \frac{\sqrt{2}}{\sqrt{\bar{\gamma}^{2}+1}} & \frac{\bar{\gamma}}{\sqrt{\bar{\gamma}^{2}+1}} \\
\frac{-\bar{\gamma}}{\sqrt{\bar{\gamma}^{2}+1}} & \frac{-\sqrt{2} \bar{\gamma}}{\sqrt{\bar{\gamma}^{2}+1}} & \frac{1}{\sqrt{\bar{\gamma}^{2}+1}}
\end{array}\right)\left(\begin{array}{l}
\tilde{e} \\
\tilde{t} \\
\tilde{g}
\end{array}\right) \text { ii) }\left(\begin{array}{l}
\tilde{e}_{2} \\
\tilde{t}_{2} \\
\tilde{g}_{2}
\end{array}\right)=\left(\begin{array}{ccc}
1 & \sqrt{2} & 0 \\
\frac{\sqrt{2}}{\sqrt{\left|2 \bar{\gamma}^{2}-1\right|}} & \frac{1}{\sqrt{\left|2 \bar{\gamma}^{2}-1\right|}} & \frac{\sqrt{2} \bar{\gamma}}{\sqrt{\left|2 \bar{\gamma}^{2}-1\right|}} \\
\frac{2 \bar{\gamma}}{\sqrt{\left|2 \bar{\gamma}^{2}-1\right|}} & \frac{\sqrt{2} \bar{\gamma}}{\sqrt{\left|2 \bar{\gamma}^{2}-1\right|}} & \frac{1}{\sqrt{\left|2 \bar{\gamma}^{2}-1\right|}}
\end{array}\right)\left(\begin{array}{c}
\tilde{e} \\
\tilde{t} \\
\tilde{g}
\end{array}\right)
$$

where $\bar{\gamma}$ is as given in (2).

Proof. i) Let us investigate the dual Darboux frame fields of dual Smarandache $\tilde{e} \tilde{t}$-curve according to $\tilde{\alpha}=\tilde{\alpha}(\bar{s})$. Since $\tilde{\alpha}_{1}=\tilde{e}_{1}$, we have

$$
\tilde{e}_{1}=\sqrt{2} \tilde{e}+\tilde{t}
$$


Differentiating (8) with respect to $\bar{s}$, we get

$$
\frac{d \tilde{e}_{1}}{d \bar{s}}=\frac{d \tilde{e}_{1}}{d \bar{s}_{1}} \cdot \frac{d \bar{s}_{1}}{d \bar{s}}=\tilde{t}_{1} \cdot \frac{d \bar{s}_{1}}{d \bar{s}}=\tilde{e}+\sqrt{2} \tilde{t}+\bar{\gamma} \tilde{g}
$$

and hence

$$
\tilde{t}_{1}=\frac{1}{\sqrt{\bar{\gamma}^{2}+1}}(\tilde{e}+\sqrt{2} \tilde{t}+\bar{\gamma} \tilde{g})
$$

where $\frac{d \bar{s}_{1}}{d \bar{s}}=\sqrt{\bar{\gamma}^{2}+1}$. Thus, since $-\tilde{g}_{1}=\tilde{e}_{1} \times \tilde{t}_{1}$, we have

$$
\tilde{g}_{1}=\frac{-\bar{\gamma}}{\sqrt{\bar{\gamma}^{2}+1}} \tilde{e}-\frac{\sqrt{2} \bar{\gamma}}{\sqrt{\bar{\gamma}^{2}+1}} \tilde{t}+\frac{1}{\sqrt{\bar{\gamma}^{2}+1}} \tilde{g}
$$

From (8)-(10) we have (7).

The proof of the statement (ii) can be given by the same way of the proof of statement (i).

Theorem 4.2. The relationships between the dual Darboux formulae of dual Smarandache $\tilde{e} \tilde{t}$-curves and dual Darboux frame of $\tilde{\alpha}$ are as follows

$$
\begin{aligned}
& \text { i) }\left(\begin{array}{l}
\frac{d \tilde{e}_{1}}{d \bar{s}_{1}} \\
\frac{d \tilde{t}_{1}}{d \bar{s}_{1}} \\
\frac{d \tilde{g}_{1}}{d \bar{s}_{1}}
\end{array}\right)=\left(\begin{array}{ccc}
\frac{1}{\sqrt{\bar{\gamma}^{2}+1}} & \frac{\sqrt{2}}{\sqrt{\bar{\gamma}^{2}+1}} & \frac{\bar{\gamma}}{\sqrt{\bar{\gamma}^{2}+1}} \\
\frac{\sqrt{2}+\sqrt{2} \bar{\gamma}^{2}-\overline{\gamma \gamma}}{\left(\bar{\gamma}^{2}+1\right)^{2}} & \frac{1-\bar{\gamma}^{4}-\sqrt{2} \overline{\gamma \gamma}}{\left(\bar{\gamma}^{2}+1\right)^{2}} & \frac{\sqrt{2} \bar{\gamma}+\sqrt{2} \bar{\gamma}^{3}+\left(\bar{\gamma}^{2}-\bar{\gamma}+1\right) \bar{\gamma}}{\left(\bar{\gamma}^{2}+1\right)^{2}} \\
\frac{-\sqrt{2} \bar{\gamma}-\sqrt{2} \bar{\gamma}^{3}-\bar{\gamma}^{\prime}}{\left(\bar{\gamma}^{2}+1\right)^{2}} & \frac{-2 \bar{\gamma}-2 \bar{\gamma}^{3}-\sqrt{2} \bar{\gamma}^{\prime}}{\left(\bar{\gamma}^{2}+1\right)^{2}} & \frac{-\sqrt{2} \bar{\gamma}^{2}-\sqrt{2} \bar{\gamma}^{4}-\overline{\gamma \gamma}^{\prime}}{\left(\bar{\gamma}^{2}+1\right)^{2}}
\end{array}\right)\left(\begin{array}{l}
\tilde{e} \\
\tilde{t} \\
\tilde{g}
\end{array}\right) \\
& \text { ii) }\left(\begin{array}{l}
\frac{d \tilde{e}_{2}}{d \bar{s}_{2}} \\
\frac{d \tilde{t}_{2}}{d \bar{s}_{2}} \\
\frac{d \tilde{g}_{2}}{d \bar{s}_{2}}
\end{array}\right)=\left(\begin{array}{ccc}
\frac{\sqrt{2}}{\sqrt{2 \bar{\gamma}^{2}-1 \mid}} & \frac{1}{\sqrt{2 \bar{\gamma}^{2}-1 \mid}} \\
\frac{2 \bar{\gamma}^{2}-2 \sqrt{2} \bar{\gamma}^{\prime}-1}{\left(2 \bar{\gamma}^{2}-1\right)^{2}} & \frac{2 \bar{\gamma}+3 \sqrt{2} \bar{\gamma}^{2}-2 \sqrt{2} \bar{\gamma}^{4}-\sqrt{2}}{\left(2 \bar{\gamma}^{2}-1\right)^{2}} & \frac{2 \bar{\gamma}^{3}-\bar{\gamma}-\sqrt{2} \bar{\gamma}^{\prime}}{\left(2 \bar{\gamma}^{2}-1\right)^{2}} \\
\frac{2 \sqrt{2} \bar{\gamma}^{3}-\sqrt{2} \bar{\gamma}-2 \bar{\gamma}^{\prime}}{\left(2 \bar{\gamma}^{2}-1\right)^{2}} & \frac{2 \bar{\gamma}^{3}-\bar{\gamma}-\sqrt{2} \bar{\gamma}^{\prime}}{\left(2 \bar{\gamma}^{2}-1\right)^{2}} & \frac{2 \sqrt{2} \bar{\gamma}^{4}-\sqrt{2} \bar{\gamma}^{2}-2 \overline{\gamma \gamma}^{\prime}}{\left(2 \bar{\gamma}^{2}-1\right)^{2}}
\end{array}\right)\left(\begin{array}{l}
\tilde{e} \\
\tilde{t} \\
\tilde{g}
\end{array}\right)
\end{aligned}
$$

Proof. i) Differentiating (8), (9) and (10) with respect to $\bar{s}$, we have the desired equation (11).

The proof of the statement (ii) can be given by the same way of the proof of statement (i). 
Theorem 4.3. Let $\tilde{\alpha}=\tilde{\alpha}(\bar{s})$ be a unit speed regular curve on dual unit hyperbolic sphere. Then the relationships between the dual curvatures of $\tilde{\alpha}$ and its dual Smarandache $\tilde{e} \tilde{t}$-curves are given by
i) $\bar{\gamma}_{1}=\frac{\bar{\gamma}^{\prime}+\sqrt{2} \bar{\gamma}+\sqrt{2} \bar{\gamma}^{3}}{\left(\bar{\gamma}^{2}+1\right)^{3 / 2}}$
ii) $\bar{\gamma}_{2}=\frac{\sqrt{2} \bar{\gamma}^{\prime}+\bar{\gamma}-2 \bar{\gamma}^{3}}{\left(2 \bar{\gamma}^{2}-1\right)^{3 / 2}}$

Proof. i) Since $\frac{d \tilde{g}_{1}}{d \bar{s}_{1}}=-\bar{\gamma}_{1} \tilde{t}_{1}$, from (9) and (11), we get dual curvature of the curve $\tilde{\alpha}_{1}\left(\bar{s}_{1}\right)$ as follows

$$
\bar{\gamma}_{1}=\frac{\bar{\gamma}^{\prime}+\sqrt{2} \bar{\gamma}+\sqrt{2} \bar{\gamma}^{3}}{\left(\bar{\gamma}^{2}+1\right)^{3 / 2}}
$$

The proof of the statement (ii) can be given by the same way of the proof of statement (i).

Corollary 4.1. The instantaneous Darboux vector of dual Smarandache $\tilde{e} \tilde{t}$-curves are given by
i) $\tilde{d}_{1}=\left(\frac{\sqrt{2} \bar{\gamma}^{\prime}+3 \bar{\gamma}+3 \bar{\gamma}^{3}}{\left(1+\bar{\gamma}^{2}\right)^{3 / 2}}\right) \tilde{e}+\left(\frac{\vec{\gamma}+2 \sqrt{2} \bar{\gamma}+2 \sqrt{2} \bar{\gamma}^{3}}{\left(1+\bar{\gamma}^{2}\right)^{3 / 2}}\right) \tilde{t}+\left(\frac{1+\bar{\gamma}^{2}}{\left(1+\bar{\gamma}^{2}\right)^{3 / 2}}\right) \tilde{g}$
ii) $\tilde{d}_{2}=\left(\frac{-\sqrt{2} \bar{\gamma}^{\prime}+\bar{\gamma}-2 \bar{\gamma}^{3}}{\left(2 \bar{\gamma}^{2}-1\right)^{3 / 2}}\right) \tilde{e}+\left(\frac{-2 \bar{\gamma}^{\prime}}{\left(2 \bar{\gamma}^{2}-1\right)^{3 / 2}}\right) \tilde{t}+\left(\frac{-1}{\left(2 \bar{\gamma}^{2}-1\right)^{3 / 2}}\right) \tilde{g}$.

Proof: i) It is known that the dual instantaneous Darboux vector of dual Smarandache $\tilde{e} \tilde{t}$-curve is $\tilde{d}_{1}=-\bar{\gamma}_{1} \tilde{e}_{1}-\tilde{g}_{1}$. Then, from (8), (10) and (12) we have (13).

The proof of the statement (ii) can be given by the same way of the proof of statement (i).

Theorem 4.4. Let $\tilde{\alpha}(\bar{s})=\tilde{\alpha}$ be a unit speed regular dual curve on dual unit hyperbolic sphere. If the ruled surface corresponding to dual hyperbolic curve $\tilde{\alpha}$ is developable then the ruled surfaces corresponding to dual Smarandache curves are also developable if and only if

i) $\delta_{1}=\frac{-3 \gamma \delta\left(\gamma^{\prime}+\sqrt{2} \gamma+\sqrt{2} \gamma^{3}\right)}{\left(1+\gamma^{2}\right)^{5 / 2}}+\frac{\delta\left(\sqrt{2}+3 \gamma^{2}\right)+\delta^{\prime}}{\left(1+\gamma^{2}\right)^{3 / 2}} \quad$ ii) $\delta_{2}=\frac{-6 \gamma \delta\left(\sqrt{2} \gamma^{\prime}+\gamma-2 \gamma^{3}\right)}{\left(2 \gamma^{2}-1\right)^{5 / 2}}+\frac{\delta\left(1-6 \gamma^{2}\right)+\sqrt{2} \delta^{\prime}}{\left(2 \gamma^{2}-1\right)^{3 / 2}}$.

Proof. i) From (2) we have 


$$
\bar{\gamma}=\gamma+\varepsilon(\delta+\gamma \Delta) \quad \bar{\gamma}_{1}=\gamma_{1}+\varepsilon\left(\delta_{1}+\gamma_{1} \Delta_{1}\right)
$$

Then substituting these equalities into to equation (12) and separating its real and dual components, we have

$$
\gamma_{1}+\varepsilon\left(\delta_{1}+\gamma_{1} \Delta_{1}\right)=\frac{\gamma^{\prime}+\sqrt{2} \gamma+\sqrt{2} \gamma^{3}}{\left(1+\gamma^{2}\right)^{3 / 2}}+\varepsilon\left[\frac{-3 \gamma(\delta+\gamma \Delta)\left(\gamma^{\prime}+\sqrt{2} \gamma+\sqrt{2} \gamma^{3}\right)}{\left(1+\gamma^{2}\right)^{5 / 2}}+\frac{(\delta+\gamma \Delta)\left(\sqrt{2}+3 \gamma^{2}\right)+\delta^{\prime}+\gamma^{\prime} \Delta+\gamma \Delta^{\prime}}{\left(1+\gamma^{2}\right)^{3 / 2}}\right]
$$

Since the ruled surface corresponding to dual hyperbolic curve $\tilde{\alpha}$ is developable, $\Delta=0$. Hence,

$$
\gamma_{1}+\varepsilon\left(\delta_{1}+\gamma_{1} \Delta_{1}\right)=\frac{-3 \gamma \delta\left(\gamma^{\prime}+\sqrt{2} \gamma+\sqrt{2} \gamma^{3}\right)}{\left(1+\gamma^{2}\right)^{5 / 2}}+\frac{\delta\left(\sqrt{2}+3 \gamma^{2}\right)+\delta^{\prime}}{\left(1+\gamma^{2}\right)^{3 / 2}} .
$$

Thus, the ruled surfaces corresponding to dual Smarandache curves are developable if and only if

$$
\delta_{1}=\frac{-3 \gamma \delta\left(\gamma^{\prime}+\sqrt{2} \gamma+\sqrt{2} \gamma^{3}\right)}{\left(1+\gamma^{2}\right)^{5 / 2}}+\frac{\delta\left(\sqrt{2}+3 \gamma^{2}\right)+\delta^{\prime}}{\left(1+\gamma^{2}\right)^{3 / 2}}
$$

The proof of the statement (ii) can be given by the same way of the proof of statement (i).

Theorem 4.5. The relationships between the radius of dual curvature of dual Smarandache $\tilde{e} \tilde{t}$-curves and the dual curvature of $\tilde{\alpha}$ are given by

$$
\text { i) } \bar{R}_{1}=\frac{\left(\bar{\gamma}^{2}+1\right)^{3 / 2}}{\sqrt{\left|\left(\bar{\gamma}^{2}+1\right)^{3}-\left(\bar{\gamma}+\sqrt{2} \bar{\gamma}+\sqrt{2} \bar{\gamma}^{3}\right)^{2}\right|}} \text { ii) } \bar{R}_{2}=\frac{\left|\left(2 \bar{\gamma}^{2}-1\right)^{3 / 2}\right|}{\sqrt{\left|\left(2 \bar{\gamma}^{2}-1\right)^{3}-\left(\sqrt{2} \bar{\gamma}^{\prime}+\bar{\gamma}+2 \bar{\gamma}^{3}\right)^{2}\right|}} \text {. }
$$

Proof. i) Substituting equation (12) into to equation (3), the radius of dual curvature is

$$
\bar{R}_{1}=\frac{1}{\sqrt{\left|1-\left(\frac{\bar{\gamma}^{\prime}+\sqrt{2} \bar{\gamma}+\sqrt{2} \bar{\gamma}^{3}}{\left(\bar{\gamma}^{2}+1\right)^{3 / 2}}\right)^{2}\right|}}=\frac{\left(\bar{\gamma}^{2}+1\right)^{3 / 2}}{\sqrt{\left|\left(\bar{\gamma}^{2}+1\right)^{3}-\left(\bar{\gamma}^{\prime}+\sqrt{2} \bar{\gamma}+\sqrt{2} \bar{\gamma}^{3}\right)^{2}\right|}}
$$

Then, $\bar{R}_{1}=\frac{\left(\bar{\gamma}^{2}+1\right)^{3 / 2}}{\sqrt{\left|\left(\bar{\gamma}^{2}+1\right)^{3}-\left(\bar{\gamma}^{\prime}+\sqrt{2} \bar{\gamma}+\sqrt{2} \bar{\gamma}^{3}\right)^{2}\right|}}$.

The proof of the statement (ii) can be given by the same way of the proof of statement (i). 
In the following sections we define dual Smarandache $\tilde{t} \tilde{g}, \tilde{e} \tilde{g}$ and $\tilde{e} \tilde{t} \tilde{g}$ curves. The proofs of the theorems and corollaries of these sections can be given by using the similar way used in previous section.

\subsection{Dual Smarandache $\tilde{t} \tilde{g}$-curves of a curve lying on $\tilde{H}_{0}^{2}$}

Definition 4.2. Let $\tilde{\alpha}(\bar{s})=\tilde{\alpha}$ be a unit speed regular dual hyperbolic curve lying fully on dual unit hyperbolic sphere and $\{\tilde{e}, \tilde{t}, \tilde{g}\}$ be its moving Darboux frame. The dual curve $\tilde{\alpha}_{3}$ defined by

$$
\tilde{\alpha}_{3}=\frac{1}{\sqrt{2}}(\tilde{t}+\tilde{g})
$$

is called the dual Smarandache $\tilde{t} \tilde{g}$-curve of $\tilde{\alpha}$ and fully lies on $\tilde{S}_{1}^{2}$. Then the ruled surface corresponding to $\tilde{\alpha}_{3}$ is called the Smarandache $\tilde{t} \tilde{g}$-ruled surface of the surface corresponding to dual hyperbolic curve $\tilde{\alpha}$.

Now we can give the relationships between $\tilde{\alpha}$ and its dual Smarandache $\tilde{t} \tilde{g}$-curve $\tilde{\alpha}_{3}$ as follows.

Theorem 4.7. Let $\tilde{\alpha}(\bar{s})=\tilde{\alpha}$ be a unit speed regular dual hyperbolic curve lying on dual unit hyperbolic sphere $\tilde{H}_{0}^{2}$. Then the relationships between the dual Darboux frames of $\tilde{\alpha}$ and its dual Smarandache $\tilde{t} \tilde{g}$ -curve $\tilde{\alpha}_{3}$ are given by

$$
\left(\begin{array}{c}
\tilde{e}_{3} \\
\tilde{t}_{3} \\
\tilde{g}_{3}
\end{array}\right)=\left(\begin{array}{ccc}
0 & \frac{1}{\sqrt{2}} & \frac{1}{\sqrt{2}} \\
\frac{1}{\sqrt{\left|2 \bar{\gamma}^{2}-1\right|}} & \frac{\bar{\gamma}}{\sqrt{\left|2 \bar{\gamma}^{2}-1\right|}} & \frac{\bar{\gamma}}{\sqrt{\left|2 \bar{\gamma}^{2}-1\right|}} \\
\frac{-2 \bar{\gamma}}{\sqrt{\left|4 \bar{\gamma}^{2}-2\right|}} & \frac{1}{\sqrt{\left|4 \bar{\gamma}^{2}-2\right|}} & \frac{-1}{\sqrt{\left|4 \bar{\gamma}^{2}-2\right|}}
\end{array}\right) .\left(\begin{array}{l}
\tilde{e} \\
\tilde{t} \\
\tilde{g}
\end{array}\right) .
$$

Theorem 4.8. Let $\tilde{\alpha}(\bar{s})=\tilde{\alpha}$ be a unit speed regular dual hyperbolic curve on dual unit hyperbolic sphere $\tilde{H}_{0}^{2}$. Then according to dual Darboux frame of $\tilde{\alpha}$, the dual Darboux formulae of dual Smarandache $\tilde{t} \tilde{g}$-curve $\tilde{\alpha}_{3}$ are as follows 


$$
\left(\begin{array}{l}
\frac{d \tilde{e}_{3}}{d s_{3}} \\
\frac{d \tilde{t}_{3}}{d s_{3}} \\
\frac{d \tilde{g}_{3}}{d s_{3}}
\end{array}\right)=\left(\begin{array}{ccc}
\frac{1}{\sqrt{\left|2 \bar{\gamma}^{2}-1\right|}} & \frac{\bar{\gamma}}{\sqrt{\left|2 \bar{\gamma}^{2}-1\right|}} \\
\frac{-2 \sqrt{2} \bar{\gamma}^{\prime}-2 \sqrt{2} \bar{\gamma}^{3}+\sqrt{2} \bar{\gamma}}{\left(2 \bar{\gamma}^{2}-1\right)^{2}} & \frac{3 \sqrt{2} \bar{\gamma}^{2}-2 \sqrt{2} \bar{\gamma}^{3}+\sqrt{2} \bar{\gamma}^{\prime}-\sqrt{2}}{\left(2 \bar{\gamma}^{2}-1\right)^{2}} & \frac{-2 \sqrt{2} \bar{\gamma}^{4}+\sqrt{2} \bar{\gamma}^{2}-\sqrt{2} \bar{\gamma}^{\prime}}{\left(2 \bar{\gamma}^{2}-1\right)^{2}} \\
\frac{2 \bar{\gamma}^{\prime}+2 \bar{\gamma}^{2}-1}{\left(2 \bar{\gamma}^{2}-1\right)^{2}} & \frac{\bar{\gamma}-2 \bar{\gamma}^{3}-2 \overline{\gamma \gamma}^{\prime}}{\left(2 \bar{\gamma}^{2}-1\right)^{2}} & \frac{2 \bar{\gamma}^{3}-\bar{\gamma}+2 \overline{\gamma \gamma}^{\prime}}{\left(2 \bar{\gamma}^{2}-1\right)^{2}}
\end{array}\right)\left(\begin{array}{c}
\tilde{e} \\
\tilde{t} \\
\tilde{g}
\end{array}\right)
$$

Theorem 4.9. Then the relationship between the dual curvatures of $\tilde{\alpha}$ and its dual Smarandache $\tilde{t} \tilde{g}$ curve $\tilde{\alpha}_{3}$ is given by

$$
\bar{\gamma}_{3}=\frac{1-2 \bar{\gamma}^{\prime}-2 \bar{\gamma}^{2}}{\left(2 \bar{\gamma}^{2}-1\right)^{3 / 2}}
$$

Corollary 4.2. Relationship between the dual curvature of $\tilde{\alpha}$ and Instantaneous Darboux vector of dual Smarandache $\tilde{t} \tilde{g}$-curve is given by

$$
\tilde{d}_{3}=\frac{8 \bar{\gamma}^{3}-4 \bar{\gamma}}{\left(4 \bar{\gamma}^{2}-2\right)^{3 / 2}} \tilde{e}+\frac{4 \bar{\gamma}^{\prime}}{\left(4 \bar{\gamma}^{2}-2\right)^{3 / 2}} \tilde{t}+\frac{8 \bar{\gamma}^{2}-4 \bar{\gamma}^{\prime}-4}{\left(4 \bar{\gamma}^{2}-2\right)^{3 / 2}} \tilde{g} .
$$

Theorem 4.10. Let $\tilde{\alpha}(\bar{s})=\tilde{\alpha}$ be a unit speed regular curve on dual unit hyperbolic sphere. If the ruled surface corresponding to dual hyperbolic curve $\tilde{\alpha}$ is developable then the ruled surface corresponding to dual Smarandache $\tilde{t} \tilde{g}$-curve is also developable if and only if

$$
\delta_{3}=\frac{2 \delta^{\prime}-4 \gamma \delta}{\left(2 \gamma^{2}-1\right)^{3 / 2}}-\frac{6 \gamma \delta-12 \gamma \gamma^{\prime} \delta-12 \gamma^{3} \delta}{\left(2 \gamma^{2}-1\right)^{5 / 2}}
$$

Theorem 4.11. The relationship between the radius of dual curvature of dual Smarandache $\tilde{\text { tg }} \tilde{g}$-curves and the dual curvature of $\tilde{\alpha}$ is given by

$$
\bar{R}_{3}=\frac{\left|\left(2 \bar{\gamma}^{2}-1\right)^{3 / 2}\right|}{\sqrt{\left(2 \bar{\gamma}^{2}-1\right)^{3}-\left(1-2 \bar{\gamma}^{\prime}-2 \bar{\gamma}^{2}\right)^{2} \mid}} .
$$




\subsection{Dual Smarandache $\tilde{e} \tilde{g}$-curves of a curve lying on $\tilde{H}_{0}^{2}$}

Definition 4.3. Let $\tilde{\alpha}(\bar{s})=\tilde{\alpha}$ be a unit speed regular curve lying fully on dual unit hyperbolic sphere $\tilde{H}_{0}^{2}$ and $\{\tilde{e}, \tilde{t}, \tilde{g}\}$ be its moving Darboux frame. The dual Smarandache curves defined by
i) $\tilde{\alpha}_{4}=\sqrt{2} \tilde{e}+\tilde{g}$
ii) $\tilde{\alpha}_{5}=\tilde{e}+\sqrt{2} \tilde{g}$

are called the dual Smarandache $\tilde{e} \tilde{g}$-curves of hyperbolic curve $\tilde{\alpha} . \tilde{\alpha}_{4}$ and $\tilde{\alpha}_{5}$ fully lies on $\tilde{H}_{0}^{2}$ and $\tilde{S}_{1}^{2}$ , respectively.

Now we can give the relationships between $\tilde{\alpha}$ and its dual Smarandache $\tilde{e} \tilde{g}$-curves as follows.

Theorem 4.13. Let $\tilde{\alpha}(\bar{s})=\tilde{\alpha}$ be a unit speed regular curve lying on dual unit hyperbolic sphere $\tilde{H}_{0}^{2}$. Then the relationships between the dual Darboux frames of $\tilde{\alpha}$ and its dual Smarandache $\tilde{e} \tilde{g}$-curves are given by
i) $\left(\begin{array}{l}\tilde{e}_{4} \\ \tilde{t}_{4} \\ \tilde{g}_{4}\end{array}\right)=\left(\begin{array}{ccc}\sqrt{2} & 0 & 1 \\ 0 & 1 & 0 \\ 1 & 0 & \sqrt{2}\end{array}\right)\left(\begin{array}{l}\tilde{e} \\ \tilde{t} \\ \tilde{g}\end{array}\right)$
ii) $\left(\begin{array}{l}\tilde{e}_{5} \\ \tilde{t}_{5} \\ \tilde{g}_{5}\end{array}\right)=\left(\begin{array}{ccc}1 & 0 & \sqrt{2} \\ 0 & 1 & 0 \\ -\sqrt{2} & 0 & -1\end{array}\right)\left(\begin{array}{c}\tilde{e} \\ \tilde{t} \\ \tilde{g}\end{array}\right)$.

Theorem 4.14. The relationships between the dual Darboux formulae of dual Smarandache $\tilde{e} \tilde{g}$-curves and dual Darboux frame of $\tilde{\alpha}$ are given by

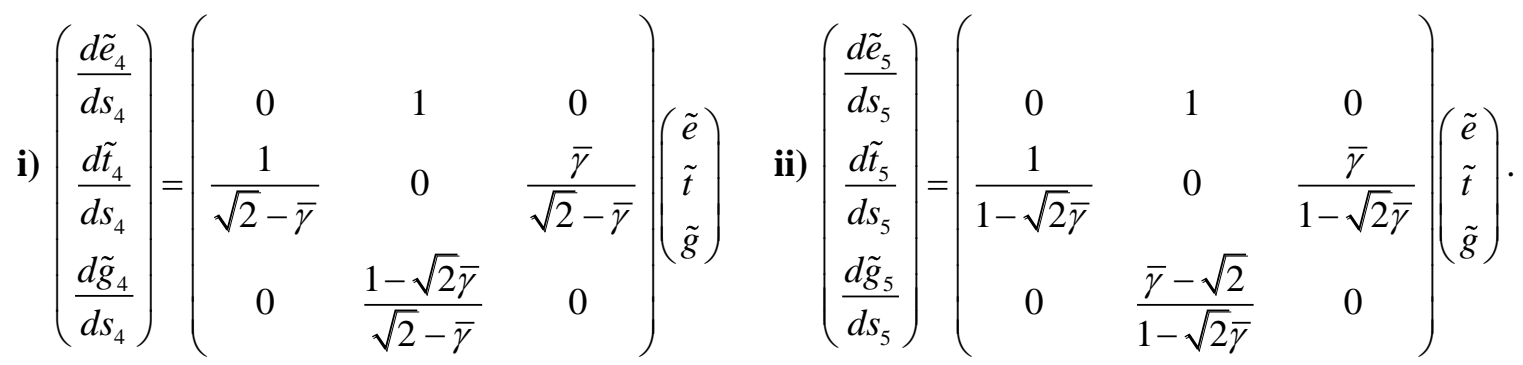

Theorem 4.15. Let $\tilde{\alpha}(\bar{s})=\tilde{\alpha}$ be a unit speed regular curve on dual unit hyperbolic sphere. Then the relationships between the dual curvatures of $\tilde{\alpha}$ and its dual Smarandache $\tilde{e} \tilde{g}$-curves are given by
i) $\bar{\gamma}_{4}=\frac{\sqrt{2} \bar{\gamma}-1}{\sqrt{2}-\bar{\gamma}}$
ii) $\bar{\gamma}_{5}=\frac{\sqrt{2}-\bar{\gamma}}{1-\sqrt{2} \bar{\gamma}}$. 
Corollary 4.4. The Instantaneous Darboux vectors of dual Smarandache ẽ $\tilde{g}$-curves are given by
i) $\tilde{d}_{4}=\frac{-\bar{\gamma}}{\sqrt{2}-\bar{\gamma}} \tilde{e}-\frac{1}{\sqrt{2}-\bar{\gamma}} \tilde{g}$
ii) $\tilde{d}_{5}=\frac{\bar{\gamma}}{1-\sqrt{2} \bar{\gamma}} \tilde{e}+\frac{1}{1-\sqrt{2} \bar{\gamma}} \tilde{g}$.

Theorem 4.16. Let $\tilde{\alpha}(\bar{s})=\tilde{\alpha}$ be a unit speed regular curve on dual unit hyperbolic sphere and $\tilde{\alpha}_{4}$ and $\tilde{\alpha}_{5}$ be the dual Smarandache $\tilde{e} \tilde{g}$-curves of $\tilde{\alpha}$. If the ruled surface corresponding to dual hyperbolic curve $\tilde{\alpha}$ is developable then the ruled surfaces corresponding to dual Smarandache $\tilde{e} \tilde{g}$-curves are also developable if and only if
i) $\delta_{4}=\frac{\sqrt{2} \delta}{\sqrt{2}-\gamma}+\frac{(\sqrt{2} \gamma-1) \delta}{(\sqrt{2}-\gamma)^{2}}$
ii) $\delta_{5}=\frac{\delta}{(1-\sqrt{2} \gamma)^{2}}$.

Theorem 4.17. The relationships between the radius of dual curvature of dual Smarandache ẽ $\tilde{g}$-curves and the dual curvature of $\tilde{\alpha}$ are given by
i) $\bar{R}_{4}=\frac{|\sqrt{2}-\bar{\gamma}|}{\sqrt{(\sqrt{2}-\bar{\gamma})^{2}-(\sqrt{2} \bar{\gamma}-1)^{2} \mid}}$
ii) $\bar{R}_{5}=\frac{|1-\sqrt{2} \bar{\gamma}|}{\sqrt{\left|(1-\sqrt{2} \bar{\gamma})^{2}-(\sqrt{2}-\bar{\gamma})^{2}\right|}}$.

\subsection{Dual Smarandache $\tilde{e} \tilde{t} \tilde{g}$-curves of a curve lying on $\tilde{H}_{0}^{2}$}

Definition 4.4. Let $\tilde{\alpha}(\bar{s})=\tilde{\alpha}$ be a unit speed regular dual curve lying fully on dual unit hyperbolic sphere and $\{\tilde{e}, \tilde{t}, \tilde{g}\}$ be its moving Darboux frame. The dual Smarandache curves defined by
i) $\tilde{\alpha}_{6}=\sqrt{3} \tilde{e}+\tilde{t}+\tilde{g}$
ii) $\tilde{\alpha}_{7}=\tilde{e}+\tilde{t}+\tilde{g}$

are called the dual Smarandache $\tilde{e} \tilde{t} \tilde{g}$-curves of hyperbolic curve $\tilde{\alpha} . \tilde{\alpha}_{6}$ and $\tilde{\alpha}_{7}$ fully lies on $\tilde{H}_{0}^{2}$ and $\tilde{S}_{1}^{2}$, respectively.

Now we can give the relationships between $\tilde{\alpha}$ and its dual Smarandache $\tilde{e} \tilde{t} \tilde{g}$-curves as follows. 
Theorem 4.19. Let $\tilde{\alpha}(\bar{s})=\tilde{\alpha}$ be a unit speed regular dual curve lying on dual unit hyperbolic sphere

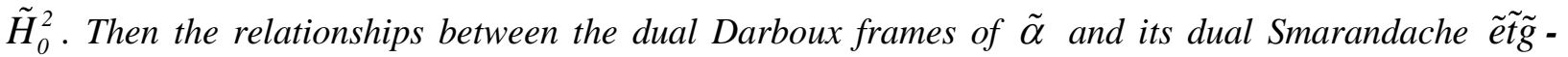
curves are given by

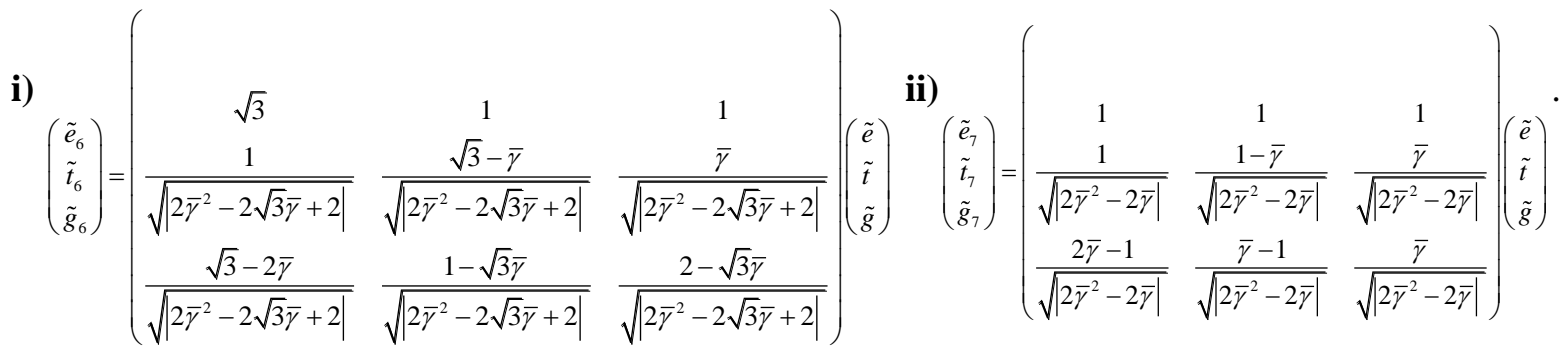

Theorem 4.20. The relationships between the dual Darboux formulae of dual Smarandache ẽ $\tilde{t} \tilde{g}$-curves and dual Darboux frame of $\tilde{\alpha}$ are given by

i)

$$
\begin{aligned}
& \left(\begin{array}{l}
\frac{d \tilde{e}_{6}}{d s_{6}} \\
\frac{d \tilde{t}_{6}}{d s_{6}} \\
\frac{d \tilde{g}_{6}}{d s_{6}}
\end{array}\right)=\left(\begin{array}{ccc}
\frac{1}{\sqrt{2 \bar{\gamma}^{2}-2 \sqrt{3} \bar{\gamma}+2 \mid}} & \frac{\sqrt{3}-\bar{\gamma}}{\sqrt{2 \bar{\gamma}^{2}-2 \sqrt{3} \bar{\gamma}+2 \mid}} \\
\frac{\sqrt{2 \bar{\gamma}^{2}-2 \sqrt{3} \bar{\gamma}+2 \mid}}{\left(2 \bar{\gamma}^{2}-2 \sqrt{3} \bar{\gamma}+2\right)^{2}} & \frac{2 \sqrt{3} \bar{\gamma}^{3}-2 \bar{\gamma}^{4}-\sqrt{3} \bar{\gamma}^{\prime}+\bar{\gamma}^{\prime}-2 \sqrt{3} \bar{\gamma}+2}{\left(2 \bar{\gamma}^{2}-2 \sqrt{3} \bar{\gamma}+2\right)^{2}} & \frac{4 \sqrt{3} \bar{\gamma}^{3}-2 \bar{\gamma}^{4}-8 \bar{\gamma}^{2}-\sqrt{3} \bar{\gamma}+2 \bar{\gamma}^{\prime}+2 \sqrt{3} \bar{\gamma}}{\left(2 \bar{\gamma}^{2}-2 \sqrt{3} \bar{\gamma}+2\right)^{2}} \\
\frac{-2 \bar{\gamma}^{2}-4 \sqrt{3} \bar{\gamma}-\bar{\gamma}^{\prime}+2}{\left(2 \bar{\gamma}^{2} \bar{\gamma}+2\right)^{2}} & \frac{\left.2 \sqrt{3} \bar{\gamma}^{4}-14 \bar{\gamma}^{3}+12 \sqrt{3} \bar{\gamma}^{2}-14 \bar{\gamma}+\bar{\gamma}\right)^{2}-\sqrt{3} \bar{\gamma}^{\prime}+2 \sqrt{3}}{(2 \sqrt{3})} & \frac{-2 \sqrt{3} \bar{\gamma}^{4}+8 \bar{\gamma}^{3}-4 \sqrt{3} \bar{\gamma}^{2}-\bar{\gamma}^{\prime}+2 \bar{\gamma}}{\left(2 \bar{\gamma}^{2}-2 \sqrt{3} \bar{\gamma}+2\right)^{2}}
\end{array}\right)\left(\begin{array}{c}
\tilde{e} \\
\tilde{t} \\
\tilde{g}
\end{array}\right) \\
& \text { ii) }\left(\begin{array}{l}
\frac{d \tilde{e}_{7}}{d s_{7}} \\
\frac{d \tilde{t}_{7}}{d s_{7}} \\
\frac{d \tilde{g}_{7}}{d s_{7}}
\end{array}\right)=\left(\begin{array}{ccc}
\frac{1}{\sqrt{\left|2 \bar{\gamma}^{2}-2 \bar{\gamma}\right|}} & \frac{\bar{\gamma}}{\sqrt{\left|2 \bar{\gamma}^{2}-2 \bar{\gamma}\right|}} \\
\frac{2 \bar{\gamma}^{\prime}-2 \bar{\gamma}^{\prime}+4 \bar{\gamma}^{2}-2 \bar{\gamma}^{3}-2 \bar{\gamma}}{\left(2 \bar{\gamma}^{2}-2 \bar{\gamma}\right)^{2}} & \frac{2 \bar{\gamma}^{2}-2 \bar{\gamma}-2 \bar{\gamma}^{4}+2 \bar{\gamma}^{3}-2 \bar{\gamma}^{\prime}+2 \bar{\gamma}^{\prime}}{\left(2 \bar{\gamma}^{2}-2 \bar{\gamma}\right)^{2}} & \frac{4 \bar{\gamma}^{3}-2 \bar{\gamma}^{4}-2 \bar{\gamma}^{2}-2 \overline{\gamma \gamma}}{\left(2 \bar{\gamma}^{2}-2 \bar{\gamma}\right)^{2}} \\
\frac{2 \bar{\gamma}^{3}-4 \bar{\gamma}^{2}+2 \bar{\gamma}-\bar{\gamma}^{\prime}}{\left(2 \bar{\gamma}^{2}-2 \bar{\gamma}\right)^{2}} & \frac{6 \bar{\gamma}^{3}-2 \bar{\gamma}^{4}-6 \bar{\gamma}^{2}+2 \bar{\gamma}+\overline{\gamma \gamma}^{\prime}-\bar{\gamma}^{\prime}}{\left(2 \bar{\gamma}^{2}-2 \bar{\gamma}\right)^{2}} & \frac{2 \bar{\gamma}^{4}-4 \bar{\gamma}^{3}+2 \bar{\gamma}^{2}-\overline{\gamma \gamma}}{\left(2 \bar{\gamma}^{2}-2 \bar{\gamma}\right)^{2}}
\end{array}\right)\left(\begin{array}{l}
\tilde{e} \\
\tilde{t} \\
\tilde{g}
\end{array}\right) .
\end{aligned}
$$

Theorem 4.21. Then the relationships between the dual curvatures of $\tilde{\alpha}$ and its dual Smarandache $\tilde{e} \tilde{t} \tilde{g}$ curves are given by
i) $\bar{\gamma}_{6}=\frac{2 \sqrt{3} \bar{\gamma}^{3}-8 \bar{\gamma}^{2}+4 \sqrt{3} \bar{\gamma}+\bar{\gamma}^{\prime}-2}{\left(2 \bar{\gamma}^{2}-2 \sqrt{3} \bar{\gamma}+2\right)^{3 / 2}}$
ii) $\bar{\gamma}_{7}=\frac{-2 \bar{\gamma}^{3}+4 \bar{\gamma}^{2}-2 \bar{\gamma}+\bar{\gamma}^{\prime}}{\left(2 \bar{\gamma}^{2}-2 \bar{\gamma}\right)^{3 / 2}}$.

Corollary 4.6. The Instantaneous Darboux vectors of dual Smarandache ẽ $\tilde{t} \tilde{g}$-curves are given by 

i) $\tilde{d}_{6}=\frac{-2 \bar{\gamma}^{3}+2 \sqrt{3} \bar{\gamma}^{2}-2 \bar{\gamma}-\sqrt{3} \bar{\gamma}^{\prime}}{\left(2 \bar{\gamma}^{2}-2 \sqrt{3} \bar{\gamma}+2\right)^{3 / 2}} \tilde{e}-\frac{\vec{\gamma}^{\prime}}{\left(2 \bar{\gamma}^{2}-2 \sqrt{3} \bar{\gamma}+2\right)^{3 / 2}} \tilde{t}+\frac{-2 \bar{\gamma}^{2}+2 \sqrt{3} \bar{\gamma}-\bar{\gamma}^{\prime}-2}{\left(2 \bar{\gamma}^{2}-2 \sqrt{3} \bar{\gamma}+2\right)^{3 / 2}} \tilde{g}$
ii) $\tilde{d}_{7}=\frac{-2 \bar{\gamma}^{3}+2 \bar{\gamma}^{2}-\vec{\gamma}}{\left(2 \bar{\gamma}^{2}-2 \bar{\gamma}\right)^{3 / 2}} \tilde{e}-\frac{\bar{\gamma}^{\prime}}{\left(2 \bar{\gamma}^{2}-2 \bar{\gamma}\right)^{3 / 2}} \tilde{t}+\frac{-2 \bar{\gamma}^{2}+2 \bar{\gamma}-\bar{\gamma}^{3}}{\left(2 \bar{\gamma}^{2}-2 \bar{\gamma}\right)^{3 / 2}} \tilde{g}$.

Theorem 4.22. Let $\tilde{\alpha}(\bar{s})=\tilde{\alpha}$ be a unit speed regular curve on dual unit hyperbolic sphere and $\tilde{\alpha}_{6}$ and $\tilde{\alpha}_{7}$ be the dual Smarandache ẽ $\tilde{t} \tilde{g}$-curves of $\tilde{\alpha}$. If the ruled surface corresponding to dual hyperbolic curve $\tilde{\alpha}$ is developable then the ruled surfaces corresponding to dual Smarandache ẽ $\tilde{t} \tilde{g}$-curves are also developable if and only if
i) $\delta_{6}=\frac{\delta\left(-2 \gamma^{3}+4 \sqrt{3} \gamma^{2}-8 \gamma-6 \gamma^{\prime}+3 \sqrt{3} \gamma^{\prime}+2 \sqrt{3}\right)}{\left(2 \gamma^{2}-2 \sqrt{3} \gamma+2\right)^{5 / 2}}+\frac{\delta^{\prime}}{\left(2 \gamma^{2}-2 \sqrt{3} \gamma+2\right)^{3 / 2}}$
ii) $\delta_{7}=\frac{\delta\left(-2 \gamma^{3}+4 \gamma^{2}-2 \gamma-6 \gamma \gamma^{\prime}+3 \gamma^{\prime}\right)}{\left(2 \gamma^{2}-2 \gamma\right)^{5 / 2}}+\frac{\delta^{\prime}}{\left(2 \gamma^{2}-2 \gamma\right)^{3 / 2}}$.

Theorem 4.23. The relationships between the radius of dual curvature of dual Smarandache ẽ $\tilde{t} \tilde{g}$-curves and the dual curvature of $\tilde{\alpha}$ are given by

$$
\begin{aligned}
& \text { i) } \bar{R}_{6}=\frac{\left|\left(2 \bar{\gamma}^{2}-2 \sqrt{3} \bar{\gamma}+2\right)^{3 / 2}\right|}{\sqrt{\left|\left(2 \bar{\gamma}^{2}-2 \sqrt{3} \bar{\gamma}+2\right)^{3}-\left(2 \sqrt{3} \bar{\gamma}^{3}-8 \bar{\gamma}^{2}+4 \sqrt{3} \bar{\gamma}+\bar{\gamma}^{\prime}-2\right)^{2}\right|}} \\
& \text { ii) } \bar{R}_{7}=\frac{\left|\left(2 \bar{\gamma}^{2}-2 \bar{\gamma}\right)^{3 / 2}\right|}{\sqrt{\left(2 \bar{\gamma}^{2}-2 \bar{\gamma}\right)^{3}-\left(-2 \bar{\gamma}^{3}+4 \bar{\gamma}^{2}-2 \bar{\gamma}+\bar{\gamma}^{\prime}\right)^{2} \mid}} .
\end{aligned}
$$

Example. Let consider the dual hyperbolic curve $\tilde{\alpha}(\bar{s})$ given by the parametrization

$$
\tilde{\alpha}(s)=\frac{1}{\sqrt{\left|1-c^{2}\right|}}[(c,-\sin s, \cos s)+\varepsilon(1,-c \sin s, c \cos s)]
$$


where $|c|>1$, particularly $c=10$. The curve $\tilde{\alpha}(s)$ represents the ruled surface

$$
r(s, v)=(10 v, \cos s-v \sin s, \sin s+v \cos s)
$$

which is a hyperboloid surface rendered in Fig. 1. Then the dual Darboux frame of $\tilde{\alpha}$ is obtained as follows,

$$
\begin{aligned}
& \tilde{e}(s)=\frac{1}{3 \sqrt{11}}[(10,-\sin 3 \sqrt{11} s, \cos 3 \sqrt{11} s)+\varepsilon(1,-10 \sin 3 \sqrt{11} s, 10 \cos 3 \sqrt{11} s)] \\
& \tilde{t}(s)=(0,-\cos 3 \sqrt{11} s,-\sin 3 \sqrt{11} s) \\
& \tilde{g}(s)=\frac{1}{3 \sqrt{11}}[(1,-10 \sin 3 \sqrt{11} s, 10 \cos 3 \sqrt{11} s)+\varepsilon(10,-\sin 3 \sqrt{11} s, \cos 3 \sqrt{11} s)] .
\end{aligned}
$$

The Smarandache $\tilde{e} \tilde{t}, \tilde{t} \tilde{g}, \tilde{e} \tilde{g}$, and $\tilde{e} \tilde{t} \tilde{g}$ curves of the dual curve $\tilde{\alpha}$ are given by

$$
\begin{aligned}
& \tilde{\alpha}_{1}(s)=\left(\frac{10 \sqrt{2}}{3 \sqrt{11}}, \frac{-\sqrt{2}}{3 \sqrt{11}} \sin 3 \sqrt{11} s-\cos 3 \sqrt{11} s, \frac{\sqrt{2}}{3 \sqrt{11}} \cos 3 \sqrt{11} s-\sin 3 \sqrt{11} s\right)+\varepsilon(1,-10 \sin 3 \sqrt{11} s, 10 \cos 3 \sqrt{11} s) \\
& \tilde{\alpha}_{2}(s)=\left(\frac{10}{3 \sqrt{11}}, \frac{-1}{3 \sqrt{11}} \sin 3 \sqrt{11} s-\sqrt{2} \cos 3 \sqrt{11} s, \frac{1}{3 \sqrt{11}} \cos 3 \sqrt{11} s-\sqrt{2} \sin 3 \sqrt{11} s\right)+\varepsilon \frac{1}{3 \sqrt{11}}(1,-10 \sin 3 \sqrt{11} s, 10 \cos 3 \sqrt{11} s) \\
& \tilde{\alpha}_{3}(s)=\left(\frac{1}{3 \sqrt{22}}, \frac{-10}{3 \sqrt{22}} \sin 3 \sqrt{11} s-\frac{\cos 3 \sqrt{11} s}{\sqrt{2}}, \frac{10}{3 \sqrt{22}} \cos 3 \sqrt{11} s-\frac{\sin 3 \sqrt{11} s}{\sqrt{2}}\right)+\varepsilon\left(5 \sqrt{2}, \frac{-\sin 3 \sqrt{11} s}{\sqrt{2}}, \frac{\cos 3 \sqrt{11} s}{\sqrt{2}}\right) \\
& \tilde{\alpha}_{4}(s)=\left(\frac{10 \sqrt{2}+1}{3 \sqrt{11}}, \frac{-(10+\sqrt{2})}{3 \sqrt{11}} \sin 3 \sqrt{11} s, \frac{10+\sqrt{2}}{3 \sqrt{11}} \cos 3 \sqrt{11} s\right)+\varepsilon\left(\frac{10+\sqrt{2}}{3 \sqrt{11}}, \frac{-(10 \sqrt{2}+1)}{3 \sqrt{11}} \sin 3 \sqrt{11} s, \frac{10 \sqrt{2}+1}{3 \sqrt{11}} \cos 3 \sqrt{11} s\right) \\
& \tilde{\alpha}_{5}(s)=\left(\frac{10+\sqrt{2}}{3 \sqrt{11}}, \frac{-(10 \sqrt{2}+1)}{3 \sqrt{11}} \sin 3 \sqrt{11} s, \frac{10 \sqrt{2}+1}{3 \sqrt{11}} \cos 3 \sqrt{11} s\right)+\varepsilon\left(\frac{10 \sqrt{2}+1}{3 \sqrt{11}}, \frac{-(10+\sqrt{2})}{3 \sqrt{11}} \sin 3 \sqrt{11} s, \frac{10+\sqrt{2}}{3 \sqrt{11}} \cos 3 \sqrt{11} s\right) \\
& \tilde{\alpha}_{6}(s)=\left(\frac{10 \sqrt{3}+1}{3 \sqrt{11}}, \frac{-(10+\sqrt{3})}{3 \sqrt{11}} \sin 3 \sqrt{11} s-\cos 3 \sqrt{11} s, \frac{10+\sqrt{3}}{3 \sqrt{11}} \cos 3 \sqrt{11} s-\sin 3 \sqrt{11} s\right)+\varepsilon\left(\frac{10+\sqrt{3}}{3 \sqrt{11}}, \frac{-(10 \sqrt{3}+1)}{3 \sqrt{11}} \sin 3 \sqrt{11} s, \frac{10 \sqrt{3}+1}{3 \sqrt{11}} \cos 3 \sqrt{11} s\right) \\
& \tilde{\alpha}_{7}(s)=\left(\frac{11}{3 \sqrt{11}}, \frac{-11}{3 \sqrt{11}} \sin 3 \sqrt{11} s-\cos 3 \sqrt{11} s, \frac{11}{3 \sqrt{11}} \cos 3 \sqrt{11} s+\sin 3 \sqrt{11} s\right)+\varepsilon\left(\frac{11}{3 \sqrt{11}}, \frac{-11}{3 \sqrt{11}} \sin 3 \sqrt{11} s, \frac{11}{3 \sqrt{11}} \cos 3 \sqrt{11} s\right) \\
& \text { respectively. From E. Study mapping, these dual spherical curves correspond to the following ruled }
\end{aligned}
$$
surfaces 


$$
\begin{aligned}
& r_{1}(s, v)=(10,-\sin 3 \sqrt{11} s, \cos 3 \sqrt{11} s)+v\left(\frac{10 \sqrt{2}}{3 \sqrt{11}}, \frac{-\sqrt{2}}{3 \sqrt{11}} \sin 3 \sqrt{11} s-\cos 3 \sqrt{11} s, \frac{\sqrt{2}}{3 \sqrt{11}} \cos 3 \sqrt{11} s-\sin 3 \sqrt{11} s\right) \\
& r_{2}(s, v)=(0, \cos 3 \sqrt{11} s, \sin 3 \sqrt{11} s)+v\left(\frac{10}{3 \sqrt{11}}, \frac{-1}{3 \sqrt{11}} \sin 3 \sqrt{11} s-\sqrt{2} \cos 3 \sqrt{11} s, \frac{1}{3 \sqrt{11}} \cos 3 \sqrt{11} s-\sqrt{2} \sin 3 \sqrt{11} s\right) \\
& r_{3}(s, v)=(1,-10 \sin 3 \sqrt{11} s, 10 \cos 3 \sqrt{11} s)+v\left(\frac{1}{3 \sqrt{22}}, \frac{-10}{3 \sqrt{11}} \sin 3 \sqrt{11} s-\frac{\cos 3 \sqrt{11} s}{\sqrt{2}}, \frac{10}{3 \sqrt{11}} \cos 3 \sqrt{11} s-\frac{\sin 3 \sqrt{11} s}{\sqrt{2}}\right) \\
& r_{4}(s, v)=(0, \cos 3 \sqrt{11} s, \sin 3 \sqrt{11} s)+v\left(\frac{10 \sqrt{2}+1}{3 \sqrt{11}}, \frac{-(10+\sqrt{2})}{3 \sqrt{11}} \sin 3 \sqrt{11} s, \frac{10+\sqrt{2}}{3 \sqrt{11}} \cos 3 \sqrt{11} s\right) \\
& r_{5}(s, v)=(0, \cos 3 \sqrt{11} s, \sin 3 \sqrt{11} s)+v\left(\frac{10+\sqrt{2}}{3 \sqrt{11}}, \frac{-(10 \sqrt{2}+1)}{3 \sqrt{11}} \sin 3 \sqrt{11} s, \frac{10 \sqrt{2}+1}{3 \sqrt{11}} \cos 3 \sqrt{11} s\right) \\
& r_{6}(s, v)=(0, \cos 3 \sqrt{11} s, \sin 3 \sqrt{11} s)+v\left(\frac{10 \sqrt{3}+1}{3 \sqrt{11}}, \frac{-(10+\sqrt{3})}{3 \sqrt{11}} \sin 3 \sqrt{11} s-\cos 3 \sqrt{11} s, \frac{10+\sqrt{3}}{3 \sqrt{11}} \cos 3 \sqrt{11} s-\sin 3 \sqrt{11} s\right) \\
& \left.r_{7}\right)=(0, \cos 3 \sqrt{11} s, \sin 3 \sqrt{11} s)+v\left(\frac{11}{3 \sqrt{11}}, \frac{-11}{3 \sqrt{11}} \sin 3 \sqrt{11} s-\cos 3 \sqrt{11} s, \frac{11}{3 \sqrt{11}} \cos 3 \sqrt{11} s+\sin 3 \sqrt{11} s\right)
\end{aligned}
$$

respectively. These surfaces are rendered in Fig.2, Fig. 3, Fig. 4 and Fig. 5, respectively.

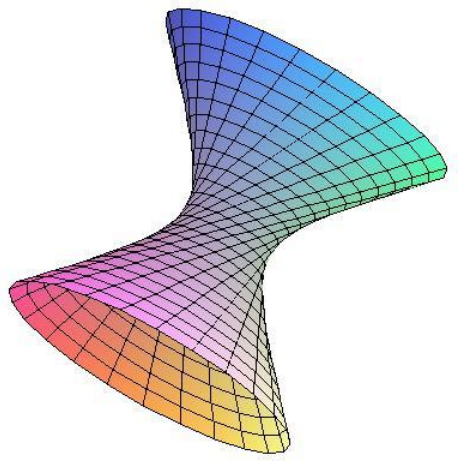

Fig.1: Hyperboloid surface corresponding to dual curve $\tilde{\alpha}$

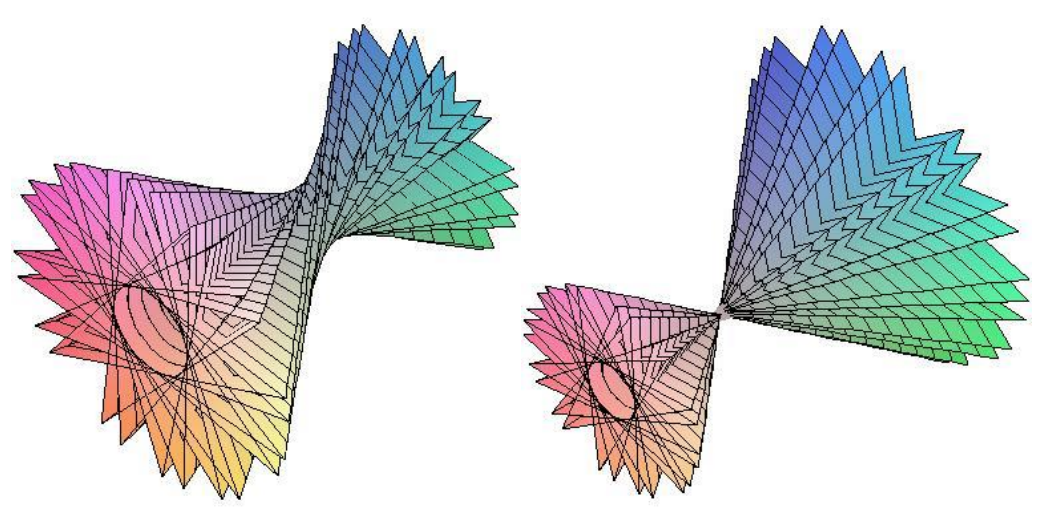

Fig.2: Smarandache $\tilde{e} \tilde{t}$ ruled surfaces 

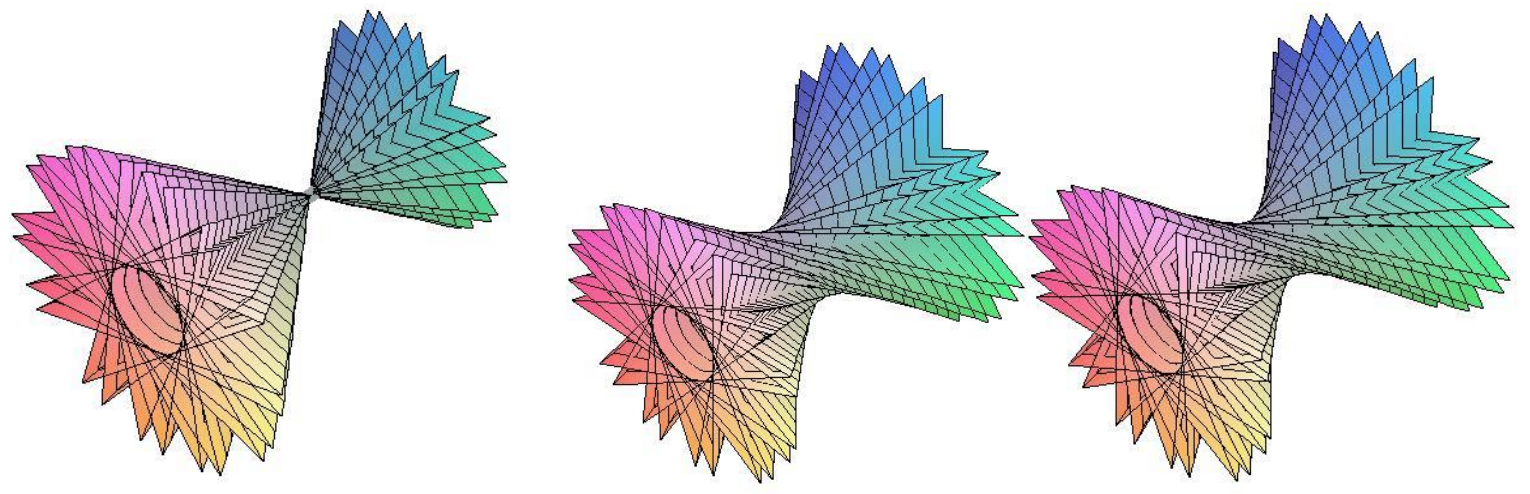

Fig.3: Smarandache $\tilde{t} \tilde{g}$ ruled surface

Fig.4: Smarandache $\tilde{e} \tilde{g}$ ruled surfaces
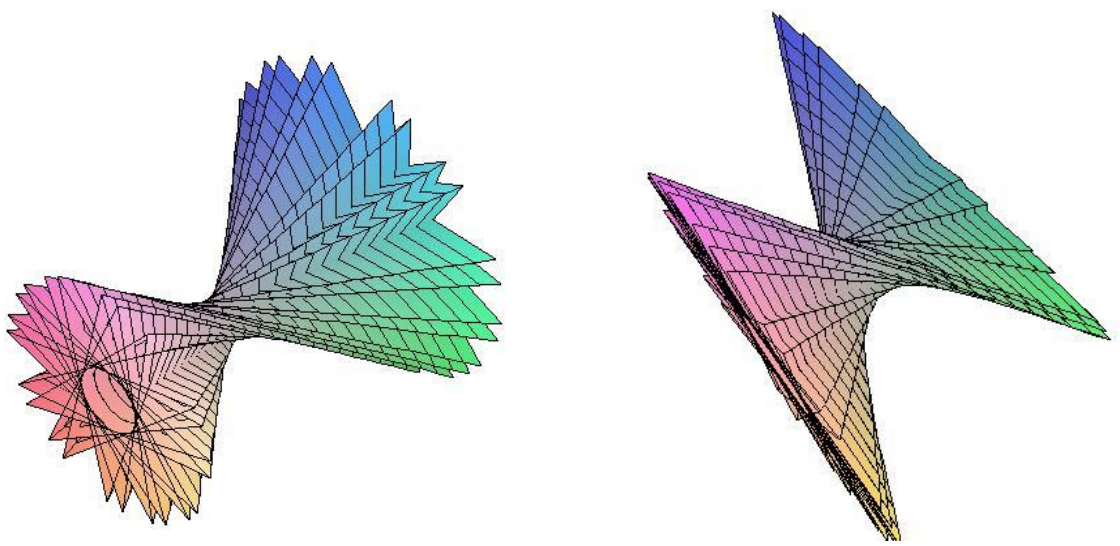

Fig.5: Smarandache $\tilde{e} \tilde{t} \tilde{g}$ ruled surfaces

Acknowledgment. We would like to thank the referees for their comments and suggestions.

\section{References}

[1] Ali, A. T., "Special Smarandache Curves in the Euclidean Space", International Journal of Mathematical Combinatorics, Vol.2 (2010), pp.30-36.

[2] Blaschke W., "Differential Geometrie and Geometrischke Grundlagen ven Einsteins Relativitasttheorie Dover". New York (1945).

[3] Dimentberg F.M., "The Screw Calculus and its Applications in Mechanics", English translation: AD680993, Clearinghouse for Federal and Scientific Technical Information, (Izdat. Nauka, Moscow, USSR) (1965).

[4] Hacısalihoğlu H.H., "Hareket Geometrisi ve Kuaterniyonlar Teorisi”, Gazi Üniversitesi Fen-Edb Fakültesi. (1983).

[5] Küçük A, Gürsoy O., "On the invariants of Bertrand trajectory surface offsets", App Math and Comp 151 (2004), 763-773.

[6] O’Neill B., "Semi-Riemannian Geometry with Applications to Relativity", Academic Press London (1983). 
[7] Önder, M., Uğurlu, H.H., "Dual Darboux Frame of a Timelike Ruled Surface and Darboux 3 Approach to Mannheim Offsets of Timelike Ruled Surfaces", Proc. Natl. Acad. Sci., India, Sect. A Phys. Sci. DOI 10.1007/s40010-013-0067-7.

[8] Taleshian, A., Asghari, N., “On Lorentzian $\alpha$-Sasakian manifold”, TJMCS Vol.4 No.3 (2012) 295-300.

[9] Turgut, M., Yilmaz, S., "Smarandache Curves in Minkowski Space-time”, Int. J. Math. Comb., 3 (2008) $51\{55\}$.

[10] Uğurlu H.H, Çalışkan A., "The Study Mapping for Directed Spacelike and Timelike Lines in Minkowski 3-Space $\mathbb{R}_{1}^{3}$ ”, Mathematical and Computational Applications 1(2) (1996),142-148.

[11] Uğurlu H.H, Çalışkan A., "Darboux Ani Dönme Vektörleri ile Spacelike ve Timelike Yüzeyler Geometrisi", Celal Bayar Üniversitesi Yayınları Yayın No: 0006 (2012).

[12] Veldkamp G.R "On the use of dual numbers, vectors and matrices in instantaneous spatial kinematics", Mechanism and Mach Theory 11 (1976), 141-156. 